\title{
Chemotherapie ist wesentlicher Therapiebestandteil beim mCRPC
}

- Steigt der PSA-Wert bei drei aufeinanderfolgenden Bestimmungen im Abstand von mindestens einer Woche an, ist die sekundäre Hormontherapie für Prostatakarzinompatienten ausgeschöpft, erklärte Prof. Udo Rebmann, Dessau. Ab diesem Zeitpunkt sind Therapien gefragt, die den klinischen Progress des laut Definition kastrationsrefraktären und in der Regel metastasierten Prostatakarzinoms (mCRPC) zumindest verlangsamen oder das Überleben verlängern, so Rebmann. Neben der Erstlinien-Chemotherapie mit Docetaxel (Taxotere ${ }^{\circledast}$ ) steht derzeit Abirateronacetat zur Verfügung, für die Zweitlinientherapie nach Versagen von Docetaxel ist Cabazitaxel (Jevtana ${ }^{\oplus}$ ) zugelassen. Auch wenn zurzeit weitere Substanzen mit verschiedenen Ansätzen entwickelt werden, sich in Prüfung befinden oder vor der Zulassung stehen, sollte laut Rebmann die Chemotherapie wesentlicher Bestandteil der onkologischen Therapieplanung bleiben. Analog zu anderen Tumorentitäten könne man postulierten, dass ein mCRPC-Patient im Krankheitsverlauf möglichst viele - im Idealfall alle - verfügbaren Therapieoptionen erhalten solle. Studien und die Versorgungsrealität zeigen, dass Patienten umso länger überleben, je früher sie aggressiv gegen den Tumor gerichtet behandelt werden.

Nach aktuellem Kenntnisstand sind Patienten mit aggressiven Tumoren (Gleason 8-10, rascher PSA-Anstieg, Schmerzen) Kandidaten für eine First-Line-Chemotherapie mit Doce- taxel. Auf der Suche nach der besseren Sequenz war die Reihenfolge Docetaxel (FirstLine) und Cabazitaxel (Second-Line) der Sequenz Docetaxel (First-Line) und Abirateron (Second-Line) im Hinblick auf die Prognose überlegen [Malik, Z et al. J Clin Oncol 2012; 30(Suppl): Abstract e15135]. Nach den Chemotherapien erreichten immerhin 54\% die Drittlinientherapie (Abirateron). Aus der Gruppe, die bereits in der First-Line mit Abirateron behandelt wurde, gelang das bei keinem.

Ute Ayazpoor

Workshop "Der Stellenwert der Chemotherapie bei mCRPC" im Rahmen der Expertise Prostata, Berlin, 2. März 2013 Veranstalter: Sanofi-Aventis, Frankfurt

\section{ED und BPS gemeinsam behandeln: erste Praxiserfahrungen gut}

- Patienten mit erektiler Dysfunktion (ED) haben häufig weitere Erkrankungen, zu denen in vielen Fällen das benigne Prostatasyndrom (BPS) gehört.,Ich erlebe in meiner Praxis viele Patienten, die mit dem Wunsch, beide Erkrankungen behandeln zu lassen, zu mir kommen", erklärt PD Dr. Tobias Engl, Oberursel, rund sechs Monate nach der erweiterten europäischen Zulassung für die Konstanztherapie mit Tadalafil (Cialis ${ }^{\circledR} 5$ mg täglich). Als erste medikamentöse Option ermöglicht sie eine gleichzeitige Behandlung von ED und BPS. So ergab eine Untersuchung, in der Tadalafil $5 \mathrm{mg}$ und

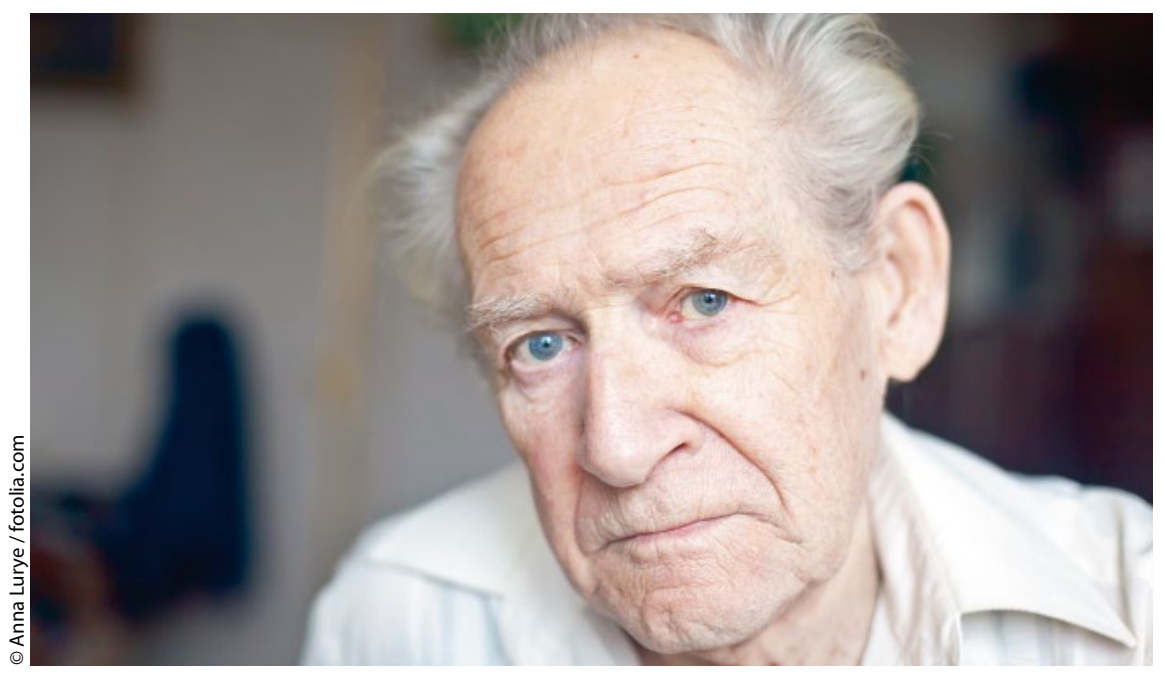

ED-Patienten haben häufig auch ein benignes Prostatasyndrom
Tamsulosin jeweils gegen Placebo getestet wurden, dass beide Wirkstoffe einen ähnlich positiven Einfluss auf das BPS haben können. NurTadalafil ermöglichte aber auch eine Steigerung der erektilen Funktion [Oelke $\mathrm{M}$ et al. Eur Urol 2012; 61: 917-25]. Die ersten Praxiserfahrungen bestätigen die guten Ergebnisse bisheriger Studien:„,Die Patienten können mit der Konstanztherapie den Geschlechtsverkehr zeitlich losgelöst von der Tabletteneinnahme genießen und mit BPS gleichzeitig ein zweites Krankheitsbild wirksam und verträglich behandeln lassen", fasst Engl zusammen. 